Research Article

\title{
On Soft Separation Axioms and Their Applications on Decision-Making Problem
}

\author{
T. M. Al-shami iD \\ Department of Mathematics, Sana'a University, Sana'a, Yemen \\ Correspondence should be addressed to T. M. Al-shami; tareqalshami83@gmail.com
}

Received 7 September 2020; Revised 24 December 2020; Accepted 31 December 2020; Published 30 January 2021

Academic Editor: Serdar Ulubeyli

Copyright (c) 2021 T. M. Al-shami. This is an open access article distributed under the Creative Commons Attribution License, which permits unrestricted use, distribution, and reproduction in any medium, provided the original work is properly cited.

\begin{abstract}
In this work, we introduce new types of soft separation axioms called $p t$-soft $\alpha$ regular and $p t$-soft $\alpha T_{i}$-spaces $(i=0,1,2,3,4)$ using partial belong and total nonbelong relations between ordinary points and soft $\alpha$-open sets. These soft separation axioms enable us to initiate new families of soft spaces and then obtain new interesting properties. We provide several examples to elucidate the relationships between them as well as their relationships with $e$-soft $T_{i}$, soft $\alpha T_{i}$, and $t t$-soft $\alpha T_{i}$-spaces. Also, we determine the conditions under which they are equivalent and link them with their counterparts on topological spaces. Furthermore, we prove that $p t$-soft $\alpha T_{i}$-spaces $(i=0,1,2,3,4)$ are additive and topological properties and demonstrate that $p t$-soft $\alpha T_{i}$-spaces $(i=0,1,2)$ are preserved under finite product of soft spaces. Finally, we discuss an application of optimal choices using the idea of $p t$-soft $T_{i}$-spaces $(i=0,1,2)$ on the content of soft weak structure. We provide an algorithm of this application with an example showing how this algorithm is carried out. In fact, this study represents the first investigation of real applications of soft separation axioms.
\end{abstract}

\section{Introduction}

The urgent need of theories dealing with uncertainties comes from daily facing complicated problems containing data which are not always crisp. The recent mathematical tool to handle these problems is soft set which was initiated by Molodtsov [1] in 1999. The rationale of soft sets is based on parameterization idea, which references that complex objects should be perceived from many aspects and each solo facet only provides a partial and approximate description of the whole entity. Molodtsov [1] in his pioneering work provided some applications of soft set in different fields and elaborated its merits compared with probability theory and fuzzy sets theory which deals with vagueness or uncertainties.

Afterwards, Maji et al. [2] started studying the operations between soft sets such as soft union and soft intersections. To overcome the shortcomings of these operations, Ali et al. [3] proposed new operations such as restricted union and intersection and a complement of a soft set and revealed some of their properties. Babitha and Sunil [4] investigated some properties of relations and functions in soft setting. Qin and Hong [5] defined new types of soft equal relations and proved some algebraic properties with respect to them. Their novel work opened up a new direction which was a goal of many papers (for more details, see [6,7] and the references mentioned therein). Recently, soft set theory has become very prevalent tool among researchers to overcome the problems of uncertainty in different fields such as information theory [8], computer sciences [9], engineering [10], and medical sciences [11].

In 2011, Shabir and Naz [12] and Çağman et al. [13] initiated a new research line by defining soft topology. However, they used two different techniques of defining soft topology. On the one hand, Shabir and Naz formulated soft topology on the collection of soft sets over a universal crisp set with a fixed set of parameters. On the other hand, Çağman et al. formulated soft topology on the collection of soft sets over an absolute soft set with different sets of parameters which are subsets of the universal set of parameters. In this paper, we continue studying soft topology using the definition given by Shabir and Naz. They formulated the 
notions of soft interior and soft closure operators and soft subspaces and shed light on soft separation axioms. Following Shabir and Naz's work, many researchers explored the topological concepts on the domain of soft topology and examined the similarity and divergence between classical topology and soft topology.

Aygünoğlu and Aygün [14] first introduced the concept of soft compactness and then Hida [15] distinguished between two types of soft compactness depending on the total belong relation. After that, Al-shami et al. [16] studied new types of soft compact and soft Lindelöf spaces. Al-shami and Kočinac [17] defined and discussed the soft version of nearly Menger spaces. Babitha and Sunil investigated some notions on soft topological spaces in [18] and presented some techniques of generated soft topology from soft relations in [19]. The authors of [20] presented soft maps by using two crisp maps, one of them between the sets of parameters and the second one between the universal sets. However, the authors of [21] introduced soft maps by using the concept of soft points.

In 2018, the authors of [22] came up new relations of belong and nonbelong between an element and soft set, namely, partial belong and total nonbelong relations. In fact, these relations widely opened the door to study and redefine many soft topological notions. This leads to obtain many fruitful properties and changes which can be seen significantly on the study of soft separation axioms and decisionmaking problems as it was shown in [23,24]. As another part of study on soft separation axioms, the authors of $[25,26]$ studied them with respect to the distinct soft points. Singh and Noorie [27] carried out a comparative study between soft separation axioms, and Terepeta [28] studied soft separating axioms and similarity of soft topological spaces. Alcantud [29] investigated the properties of countability axioms in soft setting. Recently, Al-shami $[30,31]$ has done amendments for some previous studies of soft separation axioms. For more details of conducted studies on soft set theory, we refer the reader to [32].

By decision making, we mean select the optimal alternative from the finite set of alternatives according to the multiple criteria. Decision-making theory is a very significant area, which is used mostly in human activities. In the literature, there are many studies which were conducted in this regard (see, for example, [33-35]).

The rest of this paper is organized as follows. In Section 2 , we review some basic concepts about soft sets, soft topology, and soft separation axioms. Section 3 explores the concepts $p t$-soft $\alpha T_{i}(i=0,1,2,3,4)$ and $p t$-soft $\alpha$-regular spaces using partial belong and total nonbelong relations between ordinary points and soft $\alpha$-open sets. This section shows the relationships between these concepts and discusses their main properties with the help of examples. In Section 4, we present the first investigation of real applications of soft separation axioms in decision-making problems. We initiate an algorithm of this application and show the way of carrying out this algorithm by an illustrative example. In Section 5, we discuss the advantages and limitations of the soft weak structure approach and propose some application in the engineering sciences. Finally, conclusions and some directions for future works are given in Section 6.

\section{Preliminaries}

In this section, we recall some basic concepts and properties regarding soft set theory and soft topology.

\subsection{Soft Sets}

Definition 1 (see [1]). For a nonempty set $X$ and a set of parameters $E$, a pair $(G, E)$ is said to be a soft set over $X$ provided that $G$ is a map of $E$ into the power set $P(X)$.

In this study, we use a symbol $G_{E}$ to refer a soft set instead of $(G, E)$ and we identify it as ordered pairs $G_{E}=$ $\{(e, G(e)): e \in E$ and $G(e) \in P(X)\}$.

Each $G(e)$ is called a component of $G_{E}$ (or e-approximate), and a family of all soft sets defined over $X$ with $E$ is denoted by $S\left(X_{E}\right)$.

Definition 2 (see [36]). A soft set $G_{E}$ is said to be a subset of a soft set $H_{E}$, denoted by $G_{E} \simeq H_{E}$, if $G(e) \subseteq H(e)$ for each $e \in E$.

The soft sets $G_{E}$ and $H_{E}$ are said to be soft equal if each one of them is a subset of the other.

In the literature, the relations between ordinary points and soft sets were described by four types of belong and nonbelong relations. Consequentially, new kinds of soft topological notions and concepts can be defined and studied.

Definition 3 (see $[12,22]$ ). Let $G_{E}$ be a soft set over $X$ and $x \in X$. We have the following relations:

(i) $x \in G_{E}$ if $x \in G(e)$ for each $e \in E$. We read it as $x$ totally belongs to $G_{E}$ or $G_{E}$ totally contains $x$.

(ii) $x \notin G_{E}$ if $x \notin G(e)$ for some $e \in E$. We read it as $x$ does not partially belong to $G_{E}$ or $G_{E}$ does not partially contain $x$.

(iii) $x \Subset G_{E}$ if $x \in G(e)$ for some $e \in E$. We read it as $x$ partially belongs to $G_{E}$ or $G_{E}$ partially contains $x$.

(iv) $x \notin G_{E}$ if $x \notin G(e)$ for each $e \in E$. We read it as $x$ does not totally belong to $G_{E}$ or $G_{E}$ does not totally contain $x$.

Definition 4 (see [3]). The relative complement of a soft set $G_{E}$ is a soft set $G_{E}^{c}$, where $G^{c}: E \longrightarrow 2^{X}$ is a mapping defined by $G^{c}(e)=X \backslash G(e)$ for all $e \in E$.

Two special soft sets over $X$ are the null soft set $\widetilde{\Phi}$ in which all components are the empty set and the absolute $\widetilde{X}$ soft set in which all components are the initial (universal) set $X$. Also, we say that a soft set is countable (resp. finite) if all components are countable (resp. finite). Otherwise, it is said to be uncountable (resp. infinite). Soft point is an important type of soft sets which was defined by making one of its approximations a singleton set and all the other approximations empty set. If we make all approximations of a soft set equal to a fixed subset $S$ of the universal set $X$, then we call it a stable soft set and denote it by $\widetilde{S}$. In particular, if 
$S=\{x\}$, we write $x_{E}$ instead of $\widetilde{S}$. To see the main properties of these types of soft set, we refer to [2, 22, 37, 38].

Definition 5 (see $[2,3]$ ). Let $G_{E}$ and $H_{E}$ be two soft sets over $X$.

(i) Their intersection, denoted by $G_{E} \widetilde{\cap} H_{E}$, is a soft set $U_{E}$, where a mapping $U: E \longrightarrow 2^{X}$ is given by $U(e)=G(e) \cap H(e)$.

(ii) Their union, denoted by $G_{E} \widetilde{\cup} H_{E}$, is a soft set $U_{E}$, where a mapping $U: E \longrightarrow 2^{X}$ is given by $U(e)=G(e) \cup H(e)$.

By using a similar method, the soft union and intersection operators were generalized for an arbitrary number of soft sets.

Definition 6 (see [4]). The Cartesian product of two soft sets $G_{E}$ and $H_{F}$ over $X$ and $Y$, respectively, is a soft set $G \times H_{E \times F}$ over $X \times Y$ defined by $(G \times H)(e, f)=G(e) \times H(f)$ for each $(e, f) \in E \times F$.

\subsection{Soft Topology}

Definition 7 (see [12]). A family $\tau$ of soft sets over $X$ under a fixed set of parameters $E$ is said to be a soft topology on $X$ if it satisfies the following.

(i) $\widetilde{X}$ and $\widetilde{\Phi}$ are members of $\tau$.

(ii) The intersection of a finite number of soft sets in $\tau$ is a member of $\tau$.

(iii) The union of an arbitrary number of soft sets in $\tau$ is a member of $\tau$.

The triple $(X, \tau, E)$ is called a soft topological space. A member in $\tau$ is called soft open and its relative complement is called soft closed.

Throughout this paper, $(X, \tau, E)$ and $(Y, \theta, E)$ denote soft topological spaces unless otherwise explicitly stated.

Proposition 1 (see [12]). In $(X, \tau, E)$, a family $\tau_{e}=\left\{G(e): G_{E} \in \tau\right\}$ is a classical topology on $X$ for each $e \in E . \tau_{e}$ is called a parametric topology and $\left(X, \tau_{e}\right)$ is called a parametric topological space.

Definition 8 (see [12]). Let $(X, \tau, E)$ be a soft topological space and $\varnothing \neq Y \subseteq X$. A family $\tau_{Y}=\left\{\widetilde{Y} \widetilde{\cap} t G_{E} n: q G_{E} h \epsilon_{\tau}\right\}$ is called a soft relative topology on $Y$ and the triple $\left(Y, \tau_{Y}, E\right)$ is called a soft subspace of $(X, \tau, E)$.

Definition 9 (see [39]). A subset $G_{E}$ of $(X, \tau, E)$ is called soft $\alpha$-open if $G_{E} \widetilde{\subseteq} \operatorname{int}\left(c l\left(\operatorname{int}\left(G_{E}\right)\right)\right)$.

Theorem 1 (see [39])

(i) Every soft open set is soft $\alpha$-open.

(ii) The arbitrary union (finite intersection) of soft $\alpha$-open sets is soft $\alpha$-open.
The above theorem implies that the family of all soft $\alpha$-open subsets of $(X, \tau, E)$ forms a new soft topology $\tau_{\alpha}$ finer than $\tau$.

Proposition 2. Let $\widetilde{Y}$ be soft open subset of $(X, \tau, E)$. Then:

(1) If $(H, E)$ is soft $\alpha$-open and $\tilde{Y}$ is soft open in $(X, \tau, E)$, then $(H, E) \cap(Y, E)$ is a soft $\alpha$-open subset of $\left(Y, \tau_{Y}, E\right)$.

(2) If $\tilde{Y}$ is soft open in $(X, \tau, E)$ and $(H, E)$ is a soft $\alpha$-open in $\left(Y, \tau_{Y}, E\right)$, then $(H, E)$ is a soft $\alpha$-open subset of $(X, \tau, E)$.

Definition 10 (see [40]). $(X, \tau, E)$ is said to be

(i) Soft $\alpha T_{0}$ if for every $x \neq y \in X$, there is a soft $\alpha$-open set $U_{E}$ such that $x \in U_{E}$ and $y \notin U_{E}$ or $y \in U_{E}$ and $x \notin U_{E}$.

(ii) Soft $\alpha T_{1}$ if for every $x \neq y \in X$, there are two soft $\alpha$-open sets $U_{E}$ and $V_{E}$ such that $x \in U_{E}$ and $y \notin U_{E}$ and $y \in V_{E}$ and $x \notin V_{E}$.

(iii) Soft $\alpha T_{2}$ if for every $x \neq y \in X$, there are two disjoint soft $\alpha$-open sets $U_{E}$ and $V_{E}$ such that $x \in G_{E}$ and $y \in F_{E}$.

(iv) Soft $\alpha$-regular if for every soft $\alpha$-closed set $H_{E}$ and $x \in X$ such that $x \notin H_{E}$, there are two disjoint soft $\alpha$-open sets $U_{E}$ and $V_{E}$ such that $H_{E} \widetilde{\subseteq} U_{E}$ and $x \in V_{E}$.

(v) Soft $\alpha$-normal if for every two disjoint soft $\alpha$-closed sets $H_{E}$ and $F_{E}$, there are two disjoint soft $\alpha$-open sets $U_{E}$ and $V_{E}$ such that $H_{E} \widetilde{\simeq} U_{E}$ and $F_{E} \widetilde{\subseteq} V_{E}$.

(vi) Soft $\alpha T_{3}$ (resp. soft $\alpha T_{4}$ ) if it is both soft $\alpha$-regular (resp. soft $\alpha$-normal) and soft $\alpha T_{1}$-space.

Remark 1

(i) The definitions of $e$-soft $T_{i}$-spaces $(i=0,1,2,3,4)$ of [23] were given by replacing soft $\alpha$-open and soft $\alpha$-closed sets of the above definition by soft open and soft closed sets with respect to partial belong and total nonbelong relations.

(ii) The definitions of $t t$-soft $\alpha T_{i}$-spaces $(i=0,1,2,3,4)$ of [41] were given by replacing a partial nonbelong relation of the above definition by a total nonbelong relation.

Definition 11. A soft topology $\tau$ on $X$ is said to be

(i) An enriched soft topology [14] if all soft sets $G_{E}$ such that $G(e)=\varnothing$ or $X$ are members of $\tau$.

(ii) An extended soft topology [38] if $\tau=\left\{G_{E}: G(e) \epsilon\right.$ $\tau_{e}$ for each $\left.e \in E\right\}$, where $\tau_{e}$ is a parametric topology on $X$.

The equivalence of enriched and extended soft topologies was proved in [42]. This result helps to probe the 
relationships between soft topology and its parametric topologies.

Theorem 2 (see [42]). A subset $(F, E)$ of an extended soft topological space $(X, \tau, E)$ is soft $\alpha$-open if and only if each e-approximate element of $(F, E)$ is $\alpha$-open.

Proposition 3 (see [43]). Let $\left\{\left(X_{i}, \tau_{i}, E\right): i \in I\right\}$ be a family of pairwise disjoint soft topological spaces and $X=\cup_{i \in I} X_{i}$. Then, the collection $\tau=\left\{(G, E) \widetilde{\subseteq}:(G, E) \widetilde{\cap} \widetilde{X}_{i}\right.$ which is a soft open set in $\left(X_{i}, \tau_{i}, E\right)$ for every $\left.i \in I\right\}$ defines a soft topology on $X$ with a fixed set of parameters $E$.

Definition 12 (see [43]). The soft topological space $(X, \tau, E)$ given in the above proposition is said to be the sum of soft topological spaces and is denoted by $\left(\oplus_{i \in I} X_{i}, \tau, E\right)$.

Theorem 3 (see [43]). A soft set $(G, E) \widetilde{\subseteq \widetilde{\oplus}_{i \in I} X_{i}}$ is soft $\alpha$-open (resp. soft $\alpha$-closed) in $\left(\oplus_{i \in I} X_{i}, \tau, E\right)$ if and only if all $(G, E) \cap \widetilde{X}_{i}$ are soft $\alpha$-open (resp. soft $\alpha$-closed) in $\left(X_{i}, \tau_{i}, E\right)$.

Theorem 4 (see [44]). Let $(X, \tau, A)$ and $(Y, \theta, B)$ be two soft topological spaces and $\Omega=\left\{G_{A} \times F_{B}: G_{A} \in \tau\right.$ and $\left.F_{B} \in \theta\right\}$. Then, the family of all arbitrary union of elements of $\Omega$ is a soft topology over $X \times Y$ under a fixed set of parameters $A \times B$.

Definition 13 (see [45]). A family $\tau$ of soft sets over $X$ under a fixed set of parameters $E$ is said to be a soft weak structure on $X$ if $\tau$ contains the null soft set $\widetilde{\Phi}$.

\section{3. $p t$-Soft $\alpha T_{0}$-Spaces $(i==0,1,2,3,4)$}

In this section, we define a new class of soft separation axioms called $p t$-soft $\alpha T_{0}$-spaces ( $\left.i=0,1,2,3,4\right)$, where the notations $p$ and $t$ indicate partial belong and total nonbelong relations, respectively. The initiation of this class is based on the relationship between ordinary points and soft $\alpha$-open sets with respect to partial belong and total nonbelong relations. We ascertain the relationships between them and reveal their main properties.

Definition 14. $(X, \tau, E)$ is said to be

(i) $p t$-soft $\alpha T_{0}$ if for every $x \neq y \in X$, there exists a soft $\alpha$-open set $U_{E}$ such that $x \Subset U_{E}$ and $y \notin U_{E}$ or $y \Subset U_{E}$ and $x \notin U_{E}$.

(ii) $p t$-soft $\alpha T_{1}$ if for every $x \neq y \in X$, there exist soft $\alpha$-open sets $U_{E}$ and $V_{E}$ such that $x \Subset U_{E}$ and $y \notin U_{E}$ and $y \Subset V_{E}$ and $x \notin V_{E}$.

(iii) $p t$-soft $\alpha T_{2}$ if for every $x \neq y \in X$, there exist two disjoint soft $\alpha$-open sets $U_{E}$ and $V_{E}$ such that $x \Subset U_{E}$ and $y \notin U_{E}$ and $y \Subset V_{E}$ and $x \notin V_{E}$.

(iv) $p t$-soft $\alpha$ regular if for every soft $\alpha$-closed set $H_{E}$ and $x \in X$ such that $x \notin H_{E}$, there exist disjoint soft $\alpha$-open sets $U_{E}$ and $V_{E}$ such that $H_{E} \subseteq U_{E}$ and $x \Subset V_{E}$.

(v) $p t$-soft $\alpha T_{3}$ (resp. pt-soft $\alpha T_{4}$ ) if it is both $p t$-soft $\alpha$ regular (resp. soft $\alpha$ normal) and $p t$-soft $\alpha T_{1}$.
We begin this work by showing the relationships between $p t$-soft $\alpha T_{i}$-spaces as well as their relationships with $e$-soft $T_{i}$-spaces and soft $\alpha T_{i}$-spaces.

\section{Proposition 4}

(i) Every pt-soft $\alpha T_{i}$-space is pt-soft $\alpha T_{i-1}$ for $i=0,1,2,3$.

(ii) Every e-soft $T_{i}$-space is pt-soft $\alpha T_{i}$ for $i=0,1,2,4$.

(iii) Every tt-soft $\alpha T_{i}$-space is pt-soft $\alpha T_{i}$ for $i=0,1,2,4$.

(iv) Every soft $\alpha T_{i}$-space is pt-soft $\alpha T_{i}$ for $i=2,3$.

Proof

(i) It immediately follows from Definition 16 that $p t$-soft $\alpha T_{2}$ implies $p t$-soft $\alpha T_{1}$ and $p t$-soft $\alpha T_{1}$ implies $p t$-soft $\alpha T_{0}$.

To prove that $p t$-soft $\alpha T_{3}$ implies $p t$-soft $\alpha T_{2}$, let $x \neq y$ in a $p t$-soft $\alpha T_{3}$-space $(X, \tau, E)$. Since it is $p t$-soft $\alpha T_{1}$, then there are two soft $\alpha$-open sets $U_{E}$ and $V_{E}$ such that $x \Subset U_{E}, y \notin U_{E}$ and $y \Subset V_{E}, x \notin V_{E}$. Now, $x \notin U_{E}^{c}$ and $y \notin V_{E}^{c}$. By hypothesis, $(X, \tau, E)$ is $p t$-soft regular; then, we have the following:

(1) There are two disjoint soft $\alpha$-open sets $M_{E}$ and $N_{E}$ such that $U_{E}^{c} \subseteq M_{E}$ and $x \Subset N_{E}$. Therefore, $y \in M_{E}$ and $y \notin N_{E}$.

(2) There are two disjoint soft $\alpha$-open sets $H_{E}$ and $F_{E}$ such that $V_{E}^{c} \widetilde{\subseteq} H_{E}$ and $y \Subset F_{E}$. Therefore, $x \in H_{E}$ and $x \notin F_{E}$.

From (1) and (2) above, we find that $x \Subset N_{E} \widetilde{\cap} H_{E}$, $y \notin N_{E} \widehat{\cap} H_{E}$ and $y \Subset M_{E} \widetilde{\cap} F_{E}, x \notin M_{E} \widetilde{\cap} F_{E}$. It follows from Theorem 1 that $N_{E} \widetilde{\cap} H_{E}$ and $M_{E} \widetilde{\cap} F_{E}$ are soft $\alpha$-open sets. The disjointness of them proves that $(X, \tau, E)$ is $p t$-soft $\alpha T_{2}$.

(ii) It follows from the fact that every soft $\alpha$-open set is soft open.

(iii) It follows from the fact that a total belong relation implies partial belong.

(iv) When $i=2$, let $x \neq y$ in a soft $\alpha T_{2}$-space $(X, \tau, E)$. Then, there exist two disjoint soft $\alpha$-open sets $U_{E}$ and $V_{E}$ such that $x \in U_{E}$ and $y \notin U_{E}$ and $y \in V_{E}$ and $x \notin V_{E}$. The disjointness of $U_{E}$ and $V_{E}$ leads to $y \notin U_{E}$ and $x \notin V_{E}$ as well. Thus, $(X, \tau, E)$ is $p t$-soft $\alpha T_{2}$.

When $i=3$, it is clear that a soft $\alpha$ regular space is $p t$-soft $\alpha$ regular. Also, we know that every soft $\alpha$-open and soft $\alpha$-closed subsets of a soft $\alpha$ regular space are stable. Then, a soft $\alpha T_{1}$-space is $p t$-soft $\alpha T_{1}$. Hence, a soft $\alpha T_{3}$-space is $p t$-soft $\alpha T_{3}$, as required.

The succeeding examples illustrate that the above proposition is not always reversible.

Example 1. Consider the following three soft sets defined over the universal set $X=\{x, y\}$ with a set of parameters $E=\left\{e_{1}, e_{2}\right\}$ as follows: 


$$
\begin{aligned}
& U_{1_{E}}=\left\{\left(e_{1},\{x\}\right),\left(e_{2}, \varnothing\right)\right\}, \\
& U_{2_{E}}=\left\{\left(e_{1}, \varnothing\right),\left(e_{2},\{y\}\right)\right\}, \\
& U_{3_{E}}=\left\{\left(e_{1},\{x\}\right),\left(e_{2},\{y\}\right)\right\} .
\end{aligned}
$$

Then, $\tau=\left\{\widetilde{\Phi}, t \tilde{X} n, q U_{i_{E}} h:{ }_{i} x=71,2,3\right\}$ is a soft topology on $X$. Now, $x \neq y$. One can note the following cases:

(1) $(X, \tau, E)$ is $p t$-soft $\alpha T_{2}$ because $U_{1_{E}}$ and $U_{2_{E}}$ are two disjoint soft $\alpha$-open sets partially containing $x$ and $y$, respectively, such that $x \notin U_{2_{E}}$ and $y \notin U_{1_{E}}$. In turn, $(X, \tau, E)$ is not soft $\alpha T_{2}$ because there does not exist a proper soft $\alpha$-open subset of $(X, \tau, E)$ totally containing $x$ or $y$.

(2) $(X, \tau, E)$ is soft $\alpha$ normal because the absolute and null soft sets are the only disjoint soft $\alpha$-closed subsets of $(X, \tau, E)$. Then, it is $p t$-soft $\alpha T_{4}$.

(3) $(X, \tau, E)$ is not a $t t$-soft $\alpha T_{0}$-space because there does not exist a proper soft $\alpha$-open subset of $(X, \tau, E)$ totally containing $x$ or $y$. This also means that $(X, \tau, E)$ is not $t t$-soft $\alpha T_{4}$.

(4) For a soft $\alpha$-closed set $U_{3 E}^{c}$, we have $x \notin U_{3 E}^{c}$. Since the only soft $\alpha$-open set containing $U_{3 E}^{c}$ is the absolute soft set, $(X, \tau, E)$ is not $p t$-soft $\alpha$ regular space. Consequently, it is not $p t$-soft $\alpha T_{3}$.

Example 2. Let $E$ be any set of parameters and $\tau=\left\{\widetilde{\Phi}, t U_{E} n \widetilde{\subseteq} q \mathbb{N} h:{ }_{U} E^{c} x\right.$ is finite $\}$ be a soft topology on the set of natural numbers $\mathbb{N}$. It is clear that a soft subset of $(\mathbb{N}, \tau, E)$ is soft $\alpha$-open if and only if it is soft open. For each $x \neq y \in \mathbb{N}, \widetilde{\mathbb{N} \backslash\{y}$ and $\widetilde{\mathbb{N} \backslash\{x}\}$ are soft $\alpha$-open sets such that $x \Subset \mathbb{N} \backslash\{y\}$ and $y \notin \mathbb{N} \backslash\{y\}$ and $y \Subset \mathbb{N} \backslash\{x\}$ and $x \notin \mathbb{N} \backslash\{x\}$. Therefore, $(\mathbb{N}, \tau, E)$ is $p t$-soft $\alpha T_{1}$. On the other hand, there do not exist two disjoint soft $\alpha$-open sets except for the null and absolute soft sets. Hence, $(\mathbb{N}, \tau, E)$ is not $p t$-soft $\alpha T_{2}$.

Example 3. Let $\tau=\left\{\widetilde{\Phi}, t \tilde{X} n, q\left\{\left(e_{1},\{x\}\right),\left(e_{2}, \varnothing\right)\right\}\right\}$ be a soft topology on $X=\{x, y\}$ with a set of parameters $E=\left\{e_{1}, e_{2}\right\}$. It can be easily checked that $(X, \tau, E)$ is $p t$-soft $\alpha T_{0}$, but not pt-soft $\alpha T_{1}$.

Example 4. It is well known that a soft topological space is a classical topological space if $E$ is a singleton. In this case, the concepts of $e$-soft $T_{i}$-spaces and $T_{i}$-spaces are identical, and the concepts of $p t$-soft $\alpha T_{i}$ (soft $\alpha T_{i}$ ) and $\alpha T_{i}$-spaces are identical for $i=0,1,2,3,4$. In order to show that item (ii) of the above proposition fails, we suffice by examples of classical topological spaces that satisfy an $\alpha T_{i}$-space, but not $T_{i}$ for $i=0,1,2,3,4$.

Now, we proceed to show the main properties of $p t$-soft $\alpha T_{i}$-spaces and determine the conditions under which they are equivalent.

Proposition 5. $(X, \tau, E)$ is pt-soft $\alpha T_{1}$ if $x_{E}$ is a soft $\alpha$-closed set for every $x \in X$.

Proof. For every $x \neq y, y \in\left(x_{E}\right)^{c}$ and $x \in\left(y_{E}\right)^{c}$ are soft $\alpha$-open sets. It is clear that $x \in\left(y_{E}\right)^{c}$ and $y \notin\left(y_{E}\right)^{c}$ and $y \in\left(x_{E}\right)^{c}$ and $x \notin\left(x_{E}\right)^{c}$. Hence, $(X, \tau, E)$ is pt-soft $\alpha T_{1}$.
Theorem 5. If $\left(X, \tau_{\alpha}, E\right)$ has a soft basis consisting of soft $\alpha$-clopen sets, then $(X, \tau, E)$ is pt-soft $\alpha$ regular.

Proof. Suppose that $H_{E}$ is a soft $\alpha$-closed subset of $(X, \tau, E)$ such that $x \notin H_{E}$ for some $x \in X$. Then, $H_{E}^{c}$ is a soft $\alpha$-open set such that $x \Subset H_{E}^{c}$. By hypothesis, there is a soft $\alpha$-clopen set $F_{E}$ in the basis of $\left(X, \tau_{\alpha}, E\right)$ such that $x \Subset F_{E} \widetilde{\subseteq} H_{E}^{c}$. Now, $H_{E} \subseteq F_{E}^{c}$. Obviously, $F_{E}$ and $F_{E}^{c}$ are disjoint soft $\alpha$-open sets. Hence, $(X, \tau, E)$ is $p t$-soft $\alpha$ regular.

The following results determine the condition under which $p t$-soft $\alpha T_{i}$, soft $\alpha T_{i}$, and $t t$-soft $\alpha T_{i}$-spaces are equivalent.

Theorem 6. The concepts of pt-soft $\alpha T_{i}$ and soft $\alpha T_{i}$ are equivalent for $i=0,1$ if $(X, \tau, E)$ is extended.

Proof. We prove the theorem in the case of $i=0$, as the proof of $i=1$ is analogous.

$\Rightarrow$ : Let $(X, \tau, E)$ be a soft $\alpha T_{0}$-space and let $x \neq y$. Without loss of generality, there exists a soft $\alpha$-open set $U_{E}$ such that $x \in U_{E}$ and $y \notin U_{E}$. If $y \notin U(e)$ for each $e \in E$, then the proof is trivial. So, without loss of generality, we consider there exists $e \in E$ such that $y \notin U(e)$ and $y \in U\left(e^{\prime}\right)$ for each $e^{\prime} \in E \backslash\{e\}$. Since $(X, \tau, E)$ is extended, then $U(e)$ is an $\alpha$-open subset of $\left(X, \tau_{e}\right)$. It follows from Theorem 2 that a soft set $V_{E}$ given by $V(e)=U(e)$ and $V\left(e^{\prime}\right)=\varnothing$ for each $e^{\prime} \in E \backslash\{e\}$ is a soft $\alpha$-open set. Now, we have $x \Subset V_{E}$ and $y \notin V_{E}$. Hence, $(X, \tau, E)$ is $p t$-soft $\alpha T_{0}$.

$\Leftarrow$ : Let $(X, \tau, E)$ be a $p t$-soft $\alpha T_{0}$-space and let $x \neq y$. Without loss of generality, there exists a soft $\alpha$-open set $U_{E}$ such that $x \Subset U_{E}$ and $y \notin U_{E}$. If $x \in U(e)$ for each $e \in E$, then the proof is trivial. So, without loss of generality, we consider there exists $e \in E$ such that $x \in U(e)$ and $x \notin U\left(e^{\prime}\right)$ for each $e^{\prime} \in E \backslash\{e\}$. Since $(X, \tau, E)$ is extended, then there exists a soft $\alpha$-open set $V_{E}$ such that $V(e)=U(e)$ and $V\left(e^{\prime}\right)=X$ for each $e^{\prime} \in E \backslash\{e\}$. Obviously, $x \in V_{E}$ and $y \notin V_{E}$. Hence, $(X, \tau, E)$ is soft $\alpha T_{1}$.

Corollary 1. The concepts of pt-soft $\alpha T_{4}$ and soft $\alpha T_{4}$ are equivalent if $(X, \tau, E)$ is extended.

Definition 15. $(X, \tau, E)$ is said to be $\alpha$ stable if every soft $\alpha$-open set is stable.

Theorem 7. If $(X, \tau, E)$ is $\alpha$ stable, then the concepts of pt-soft $\alpha T_{i}$, soft $\alpha T_{i}$, and tt-soft $\alpha T_{i}$-spaces are equivalent for each $i=0,1,2,3,4$.

Proof. In the case of an $\alpha$ stable space, the relations of partial belong and total belong between ordinary points and soft $\alpha$-open (soft $\alpha$-closed) sets are identical, and the relations of partial nonbelong and total nonbelong between ordinary points and soft $\alpha$-open (soft $\alpha$-closed) sets are identical too. Hence, we obtain the desired equivalences.

Corollary 2. If $(X, \tau, E)$ is a soft $\alpha$ regular space, then the concepts of pt-soft $\alpha T_{i}$, soft $\alpha T_{i}$, and tt-soft $\alpha T_{i}$-spaces are equivalent for each $i=0,1,2,3,4$. 
Interchangeability "transmission" of $p t$-soft $\alpha T_{i}$-spaces and their corresponding spaces on classical topology (which are $\alpha T_{i}$-spaces) are investigated in the following findings.

Theorem 8. Let $(X, \tau, E)$ be extended. If there exists $e \in E$ such that $\left(X, \tau_{e}\right)$ is $\alpha T_{i}$, then $(X, \tau, E)$ is pt-soft $\alpha T_{i}$ for each $i=0,1,2$.

Proof. We prove the theorem in the case of $i=2$. The other cases follow similar lines.

Let $\left(X, \tau_{e}\right)$ be $\alpha T_{2}$ and let $x \neq y \in X$. Then, there exist two disjoint $\alpha$-open subsets $M, N$ of $\left(X, \tau_{e}\right)$ containing $x$ and $y$, respectively. It follows from Theorem 2 that there are two disjoint soft $\alpha$-open subsets $U_{E}$ and $V_{E}$ of $(X, \tau, E)$ such that $U(e)=M, V(e)=N$ and $U\left(e^{\prime}\right)=V\left(e^{\prime}\right)=\varnothing$ for each $e^{\prime} \in E \backslash\{e\}$. It can be seen that $x \Subset U_{E}$ and $y \notin U_{E}$ and $y \Subset V_{E}$ and $x \notin V_{E}$. Hence, $(X, \tau, E)$ is $p t$-soft $\alpha T_{2}$.

Theorem 9. Let $(X, \tau, E)$ be extended. If all parametric topological space $\left(X, \tau_{e}\right)$ is $\alpha T_{i}$, then $(X, \tau, E)$ is pt-soft $\alpha T_{i}$ for each $i=0,1,2,3,4$.

Proof. We prove the theorem in the cases of $i=3,4$. The other cases follow from the above theorem. It suffices to prove the property of $p t$-soft regular and soft normal.

First, we prove that $(X, \tau, E)$ is $p t$-soft regular. Let $H_{E}$ be a soft $\alpha$-closed set such that $x \notin H_{E}$. Then, there exists $e \in E$ such that $x \notin H(e)$. Since $\left(X, \tau_{e}\right)$ is $\alpha$ regular, then there exist $\alpha$-open subsets $M, N$ of $\left(X, \tau_{e}\right)$ such that $x \in M$ and $H(e) \subseteq N$. It follows from Theorem 2 that there exist soft $\alpha$-open subsets $U_{E}$ and $V_{E}$ of $(X, \tau, E)$ which are defined as follows:

$$
\begin{aligned}
& U(e)=M, U\left(e^{\prime}\right)=\varnothing, \quad \text { for each } e^{\prime} \in E \backslash\{e\}, \\
& V(e)=N, V\left(e^{\prime}=X, \quad \text { for each } e^{\prime} \in E \backslash\{e\} .\right.
\end{aligned}
$$

This shows that $x \Subset U_{E}, H_{E} \widetilde{\subseteq V_{E}}$. Obviously, $U_{E}$ and $V_{E}$ are disjoint. So, $(X, \tau, E)$ is $p t$-soft $\alpha$ regular. Hence, it is pt-soft $\alpha T_{3}$.

Second, we prove that $(X, \tau, E)$ is soft $\alpha$ normal. Let $H_{E}$ and $L_{E}$ be two disjoint soft $\alpha$-closed sets. Then, $H(e)$ and $L(e)$ are two disjoint $\alpha$-closed sets for each $e \in E$. Since $\left(X, \tau_{e}\right)$ is $\alpha$ normal, then there exists two disjoint $\alpha$-open sets $M$ and $N$ such that $H(e) \subseteq M$ and $L(e) \subseteq N$. It follows from Theorem 2 that there exist soft $\alpha$-open subsets $U_{E}$ and $V_{E}$ of $(X, \tau, E)$ which are defined as follows:

$$
\begin{array}{ll}
U(e)=M, U\left(e^{\prime}\right)=\varnothing, & \text { for each } e^{\prime} \in E \backslash\{e\}, \\
V(e)=N, V\left(e^{\prime}\right)=\varnothing, & \text { for each } e^{\prime} \in E \backslash\{e\} .
\end{array}
$$

Now, $\widetilde{\cup}_{\forall e \in E} U_{E}$ and $\widetilde{\cup}_{\forall e \in E} V_{E}$ are disjoint soft $\alpha$-open sets such that $H_{E} \widetilde{\subseteq} \widetilde{U}_{\forall e \in E} U_{E}$ and $L_{E} \widetilde{\subseteq} \widetilde{U}_{\forall e \in E} V_{E}$. Thus, $(X, \tau, E)$ is soft $\alpha$ normal. Hence, it is $p t$-soft $\alpha T_{4}$.

For the sake of brevity, we present the following two theorems without proof.

Theorem 10. If $(X, \tau, E)$ is a stable (soft $\alpha$ regular), then $\left(X, \tau_{e}\right)$ is $\alpha T_{i}$ iff $(X, \tau, E)$ is pt-soft $\alpha T_{i}$ for each $i=0,1,2,3,4$.
Theorem 11. The property of being a pt-soft $\alpha T_{i}$-space is hereditary for $i=0,1,2,3$.

Now, we proceed to discuss the behaviour of $p t$-soft $\alpha T_{i}$-spaces in relation with additive and topological properties and finite product spaces.

Theorem 12. The property of being a pt-soft $\alpha T_{i}$-space is an additive property for $i=0,1,2,3,4$.

Proof. We only prove the theorem in the case of $i=4$. First, we prove that a property of $p t$-soft $\alpha T_{i}$ is additive. Let $x \neq y \in \oplus_{i \in I} X_{i}$. Then, the proof is trivial if $x$ and $y$ belong to the same $X_{i_{0}}$. Therefore, we consider there exist $i_{0} \neq j_{0} \in I$ such that $x \in X_{i_{0}}$ and $y \in X_{j_{0}}$. According to the definition of sum of soft topological spaces, we obtain that $\widetilde{X_{i_{0}}}$ and $\widetilde{X_{j_{0}}}$ are soft $\alpha$-open subsets of $\left(\oplus_{i \in I} X_{i}, \tau, E\right)$. Hence, $\left(\oplus_{i \in I} X_{i}, \tau, E\right)$ is $p t$-soft $\alpha T_{1}$.

Second, we prove a property of soft $\alpha$-normality is additive. Suppose that $F_{E}$ and $H_{E}$ are two disjoint soft $\alpha$-closed subsets of $\left(\oplus_{i \in I} X_{i}, \tau, E\right)$. Then, $F_{E} \widetilde{\cap} \widetilde{X}_{i}$ and $H_{E} \widetilde{\cap} \widetilde{X}_{i}$ are soft $\alpha$-closed in $\left(X_{i}, \tau_{i}, E\right)$ for each $i \in I$. By hypothesis, there are two disjoint soft $\alpha$-open subsets $U_{i_{E}}$ and $V_{i_{E}}$ of $\left(X_{i}, \tau_{i}, E\right)$ such that $F_{E} \widetilde{\cap} \widetilde{X}_{i} \subseteq U_{i_{E}}$ and $H_{E} \widetilde{\cap} \widetilde{X}_{i} \subseteq V_{i_{E}}$. This implies that $F_{E} \subseteq \cup_{i \varepsilon I} U_{i_{E}}, H_{E} \subseteq \cup_{i \varepsilon I} V_{i_{E}}$, and $\left[\widetilde{U}_{i \varepsilon I} U_{i_{E}}\right] \widetilde{\cap} \widetilde{U}_{i \varepsilon I} V_{i_{E}}=\widetilde{\phi}$. Hence, $\left(\oplus_{i \in I} X_{i}, \tau, E\right)$ is a soft $\alpha$-normal space.

Theorem 13. The finite product of pt-soft $\alpha T_{i}$-spaces is a pt-soft $\alpha T_{i}$-space for $i=0,1,2$.

Proof. We prove the theorem in case of $i=2$. The other cases follow similar lines.

Let $(X, \tau, E)$ and $(Y, \theta, E)$ be two $p t$-soft $\alpha T_{2}$-spaces and let $\left(x_{1}, y_{1}\right) \neq\left(x_{2}, y_{2}\right)$ in $X \times X$. Then, $x_{1} \neq x_{2}$ or $y_{1} \neq y_{2}$. Without loss of generality, let $x_{1} \neq x_{2}$. Then, there exist two disjoint soft $\alpha$-open subsets $G_{E}$ and $H_{E}$ of $(X, \tau, E)$ such that $x_{1} \Subset G_{E}$ and $x_{2} \notin G_{E}$ and $x_{2} \Subset H_{E}$ and $x_{1} \notin H_{E}$. Obviously, $G_{E} \times \widetilde{Y}$ and $H_{E} \times \widetilde{X}$ are two disjoint soft $\alpha$-open subsets $X \times Y$ such that $\left(x_{1}, y_{1}\right) \Subset G_{E} \times \tilde{Y}$ and $\left(x_{2}, y_{2}\right) \notin G_{E} \times \widetilde{Y}$ and $\left(x_{2}, y_{2}\right) \Subset$ $H_{E} \times \widetilde{Y}$ and $\left(x_{1}, y_{1}\right) \notin H_{E} \times \widetilde{Y}$. Hence, $X \times Y$ is $p t$-soft $\alpha T_{2}$.

\section{An Application of Optimization via Soft Weak Structure Using $p t$-Soft $T_{i}$-Spaces $(i==0,1,2)$}

In this section, we present an application of optimal choices using the idea of $p t$-soft $T_{i}$-spaces $(i=0,1,2)$ on the content of soft weak structure. The idea of this application is based on personality characteristics of the applicants. We construct an algorithm of this application and provide an example to demonstrate how this algorithm is carried out.

First of all, we define $p t$-soft $T_{i}$-spaces $(i=0,1,2)$ on soft weak structure in a similar way of their counterparts on topological spaces.

Definition 16. A soft weak structure $(X, \tau, E)$ is said to be

(i) $p t$-soft $\mathscr{W} T_{0}$ if for every two distinct points $x, y \in X$, there exists a $\mathscr{W}$-soft open set $G_{E}$ such that $x \Subset G_{E}$ and $y \notin G_{E}$ or $y \Subset G_{E}$ and $x \notin G_{E}$. 
(ii) $p t$-soft $\mathscr{W} T_{1}$ if for every two distinct points $x, y \in X$, there exist $\mathscr{W}$-soft open sets $G_{E}$ and $F_{E}$ such that $x \Subset G_{E}$ and $y \notin G_{E}$ and $y \Subset F_{E}$ and $x \notin F_{E}$.

(iii) $p t$-soft $\mathscr{W} T_{2}$ if for every two distinct points $x, y \in X$, there exist two disjoint $\mathscr{W}$-soft open sets $G_{E}$ and $F_{E}$ such that $x \Subset G_{E}$ and $y \notin G_{E}$ and $y \Subset F_{E}$ and $x \notin F_{E}$.

Tourism companies compete with each other to attract the customers by offering different programmes of their trip activities. One of the most important methods of promotion is the choice of places of carrying out the trip programmes. Herein, we will propose a method to help the customers to select the suitable tourism company that satisfies their options.
To this end, we consider some tourism companies will carry out their trips on the same region, and their trip programmes are distributed in some places of this region for a week. We consider the places are $X=\left\{h_{i}: i=1,2, \ldots, n\right\}$ and the trip programmes are $E=\left\{e_{i}: i=1,2, \ldots, m\right\}$, where $e_{1}$ are available places of accommodation, $e_{2}$ are available places of eating, $e_{3}$ are available places of watching cinema, $e_{4}$ are available places of watching cinema, $e_{5}$ are available places of celebrations, and so on.

The idea of this application is based on three factors: the first one is the classification of the places of trip activities as a soft set for each day. For example, let the places of trip activities on Tuesday be given as follows.

Then, we describe these activities by a soft set as follows:

$$
G_{E}=\left\{\left(e_{1},\left\{h_{4}, h_{5}, h_{6}\right\}\right),\left(e_{2},\left\{h_{1}, h_{5}\right\}\right),\left(e_{3}, \varnothing\right),\left(e_{4},\left\{h_{2}, h_{5}\right\}\right),\left(e_{5},\left\{h_{3}, h_{7}\right\}\right)\right\}
$$

Note that $G\left(e_{3}\right)=\varnothing$ does not imply any shortcoming. This case means that an activity of watching cinema is unavailable on Tuesday. This matter is reasonable because the participants of the trips need not carry out all the activities every day.

The second factor is the combination of a soft weak structure from the soft sets that represent the activities of the seven days (the whole period of the trip is a week). Then, we classify this soft weak structure in four categories: non$p t$-soft $\mathscr{W} T_{0}, p t$-soft $\mathscr{W} T_{0}, p t$-soft $\mathscr{W} T_{1}$, and $p t$-soft $\mathscr{W} T_{2}$.

The third factor is based on the personality characteristics of the customers. In this application, we classify the customers into two groups: group of many visited places without repetition as much as possible and group of few visited places with repetition. According to this classification, if the soft weak structure is non- $p t$-soft $\mathscr{W} T_{0}$, then the customer will visit many places per day. Therefore, the customer who belongs to the group of many visited places will prefer tourism company that has trip programmes satisfying this condition. On the other hand, the customer who belongs to the group of few visited places will prefer tourism company that has trip programmes not satisfying this condition. He will prefer tourism company that has trip programmes with few reiterated places. In other words, his or her optimal choice will be the weaker form of available pt-soft $\mathscr{W} T_{i}$-spaces.

To illustrate this method, we give the succeeding interesting example.

Example 5. Consider that four tourism companies A, B, C, and $\mathrm{D}$ will carry out their trips on the same region and their trip programmes are distributed in seven places of this region for a week. We consider the places are $X=\left\{h_{i}: i=1,2, \ldots, 7\right\}$ and we consider trip programmes are $E=\left\{e_{i}: i=1,2, \ldots, 5\right\}$, where $e_{1}, e_{2}, e_{3}, e_{4}$, and $e_{5}$ stand for the places of accommodation, eating, watching cinema, swimming, and celebrations, respectively.

In the succeeding four tables, we outline their trip programmes as given in their brochures.

Now, we transfer the four programmes of trips given in the above four tables to four soft weak structures as follows.

(1) The soft weak structure of programmes of trip proposed by tourism company A (given in Table 1 ) is $\tau_{A}=\left\{\widetilde{\Phi}, t G_{i E} n: q i h={ }_{1}, 2 x, 7 \ldots C, ; 7\right\}$, where $G_{i E}$ is given by

$$
\begin{aligned}
& G_{1 E}=\left\{\left(e_{1},\left\{h_{1}, h_{2}, h_{3}\right\}\right),\left(e_{2},\left\{h_{3}, h_{7}\right\}\right),\left(e_{3}, \varnothing\right),\left(e_{4},\left\{h_{2}, h_{5}\right\}\right),\left(e_{5}, \varnothing\right)\right\}, \\
& G_{2 E}=\left\{\left(e_{1},\left\{h_{6}\right\}\right),\left(e_{2},\left\{h_{6}, h_{7}\right\}\right),\left(e_{3},\left\{h_{2}\right\}\right),\left(e_{4}, \varnothing\right),\left(e_{5},\left\{h_{3}, h_{4}, h_{5}\right\}\right)\right\}, \\
& G_{3 E}=\left\{\left(e_{1},\left\{h_{4}\right\}\right),\left(e_{2},\left\{h_{4}\right\}\right),\left(e_{3},\left\{h_{1}, h_{6}\right\}\right),\left(e_{4},\left\{h_{3}, h_{6}\right\}\right),\left(e_{5},\left\{h_{7}\right\}\right)\right\}, \\
& G_{4 E}=\left\{\left(e_{1},\left\{h_{3}, h_{5}, h_{7}\right\}\right),\left(e_{2},\left\{h_{2}, h_{3}, h_{6}\right\}\right),\left(e_{3},\left\{h_{2}, h_{3}\right\}\right),\left(e_{4},\left\{h_{4}, h_{5}\right\}\right),\left(e_{5},\left\{h_{3}, h_{5}\right\}\right)\right\}, \\
& G_{5 E}=\left\{\left(e_{1},\left\{h_{2}, h_{5}\right\}\right),\left(e_{2},\left\{h_{2}, h_{5}\right\}\right),\left(e_{3}, \varnothing\right),\left(e_{4},\left\{h_{1}, h_{3}, h_{6}\right\}\right),\left(e_{5}, \varnothing\right)\right\}, \\
& G_{6 E}=\left\{\left(e_{1},\left\{h_{4}, h_{7}\right\}\right),\left(e_{2},\left\{h_{2}, h_{5}\right\}\right),\left(e_{3}, \varnothing\right),\left(e_{4}, \varnothing\right),\left(e_{5},\left\{h_{6}, h_{7}\right\}\right)\right\}, \\
& G_{7 E}=\left\{\left(e_{1},\left\{h_{6}, h_{7}\right\}\right),\left(e_{2},\left\{h_{4}\right\}\right),\left(e_{3},\left\{h_{3}, h_{4}, h_{6}\right\}\right),\left(e_{4}, \varnothing\right),\left(e_{5}, \varnothing\right)\right\} .
\end{aligned}
$$


TABLe 1: Trip programmes of tourism company A.

\begin{tabular}{|c|c|c|c|c|c|}
\hline \multirow{2}{*}{$X$} & \multicolumn{5}{|c|}{ E } \\
\hline & $e_{1}:$ accommodation & $e_{2}$ : eating & $e_{3}:$ cinema & $e_{4}:$ swimming & $e_{5}:$ celebrations \\
\hline Saturday & $\left\{h_{1}, h_{2}, h_{3}\right\}$ & $\left\{h_{3}, h_{7}\right\}$ & $\varnothing$ & $\left\{h_{2}, h_{5}\right\}$ & $\varnothing$ \\
\hline Sunday & $\left\{h_{6}\right\}$ & $\left\{h_{6}, h_{7}\right\}$ & $\left\{h_{2}\right\}$ & $\varnothing$ & $\left\{h_{3}, h_{4}, h_{5}\right\}$ \\
\hline Monday & $\left\{h_{4}\right\}$ & $\left\{h_{4}\right\}$ & $\left\{h_{1}, h_{6}\right\}$ & $\left\{h_{3}, h_{6}\right\}$ & $\left\{h_{7}\right\}$ \\
\hline Tuesday & $\left\{h_{3}, h_{5}, h_{7}\right\}$ & $\left\{h_{2}, h_{3}, h_{6}\right\}$ & $\left\{h_{2}, h_{3}\right\}$ & $\left\{h_{4}, h_{5}\right\}$ & $\left\{h_{3}, h_{5}\right\}$ \\
\hline Wednesday & $\left\{h_{2}, h_{5}\right\}$ & $\left\{h_{2}, h_{5}\right\}$ & $\varnothing$ & $\left\{h_{1}, h_{3}, h_{6}\right\}$ & $\varnothing$ \\
\hline Thursday & $\left\{h_{4}, h_{7}\right\}$ & $\left\{h_{2}, h_{5}\right\}$ & $\varnothing$ & $\varnothing$ & $\left\{h_{6}, h_{7}\right\}$ \\
\hline Friday & $\left\{h_{6}, h_{7}\right\}$ & $\left\{h_{4}\right\}$ & $\left\{h_{3}, h_{4}, h_{6}\right\}$ & $\varnothing$ & $\varnothing$ \\
\hline
\end{tabular}

(2) The soft weak structure of programmes of trip proposed by tourism company B (given in Table 2 ) is
$\tau_{B}=\left\{\widetilde{\Phi}, t G_{i E} n: q i h={ }_{1}, 2 x, 7 \ldots C, ; 7\right\}$, where $G_{i E}$ is given by

$$
\begin{aligned}
& G_{1 E}=\left\{\left(e_{1},\left\{h_{1}, h_{2}\right\}\right),\left(e_{2},\left\{h_{3}\right\}\right),\left(e_{3},\left\{h_{1}, h_{2}\right\}\right),\left(e_{4}, \varnothing\right),\left(e_{5}, \varnothing\right)\right\}, \\
& G_{2 E}=\left\{\left(e_{1},\left\{h_{7}\right\}\right),\left(e_{2},\left\{h_{7}\right\}\right),\left(e_{3}, \varnothing\right),\left(e_{4},\left\{h_{1}, h_{4}, h_{6}\right\}\right),\left(e_{5},\left\{h_{1}, h_{4}, h_{6}\right\}\right)\right\}, \\
& G_{3 E}=\left\{\left(e_{1},\left\{h_{3}, h_{4}\right\}\right),\left(e_{2},\left\{h_{1}, h_{2}\right\}\right),\left(e_{3},\left\{h_{1}, h_{3}\right\}\right),\left(e_{4},\left\{h_{3}, h_{4}, h_{6}\right\}\right),\left(e_{5}, \varnothing\right)\right\}, \\
& G_{4 E}=\left\{\left(e_{1},\left\{h_{2}, h_{3}\right\}\right),\left(e_{2},\left\{h_{4}\right\}\right),\left(e_{3},\left\{h_{1}, h_{4}, h_{5}\right\}\right),\left(e_{4},\left\{h_{2}\right\}\right),\left(e_{5}, \varnothing\right)\right\}, \\
& G_{5 E}=\left\{\left(e_{1},\left\{h_{2}, h_{4}, h_{6}\right\}\right),\left(e_{2},\left\{h_{1}\right\}\right),\left(e_{3},\left\{h_{7}\right\}\right),\left(e_{4},\left\{h_{3}, h_{5}\right\}\right),\left(e_{5}, \varnothing\right)\right\}, \\
& G_{6 E}=\left\{\left(e_{1},\left\{h_{3}\right\}\right),\left(e_{2},\left\{h_{7}\right\}\right),\left(e_{3}, \varnothing\right),\left(e_{4},\left\{h_{1}, h_{6}\right\}\right),\left(e_{5},\left\{h_{4}, h_{5}, h_{7}\right\}\right)\right\}, \\
& G_{7 E}=\left\{\left(e_{1},\left\{h_{1}, h_{4}, h_{5}\right\}\right),\left(e_{2},\left\{h_{1}, h_{4}, h_{5}\right\}\right),\left(e_{3}, \varnothing\right),\left(e_{4},\left\{h_{7}\right\}\right),\left(e_{5}, \varnothing\right)\right\} .
\end{aligned}
$$

(3) The soft weak structure of programmes of trip proposed by tourism company C (given in Table 3 ) is
$\tau_{C}=\left\{\widetilde{\Phi}, t G_{i E} n: q i h={ }_{1}, 2 x, 7 \ldots C, ; 7\right\}$, where $G_{i E}$ is given by

$$
\begin{aligned}
& G_{1 E}=\left\{\left(e_{1},\left\{h_{1}\right\}\right),\left(e_{2},\left\{h_{1}, h_{3}\right\}\right),\left(e_{3},\left\{h_{2}, h_{3}\right\}\right),\left(e_{4}, \varnothing\right),\left(e_{5}, \varnothing\right)\right\}, \\
& G_{2 E}=\left\{\left(e_{1},\left\{h_{6}, h_{7}\right\}\right),\left(e_{2},\left\{h_{4}, h_{7}\right\}\right),\left(e_{3}, \varnothing\right),\left(e_{4},\left\{h_{4}, h_{5}\right\}\right),\left(e_{5},\left\{h_{4}, h_{5}\right\}\right)\right\}, \\
& G_{3 E}=\left\{\left(e_{1},\left\{h_{1}, h_{2}, h_{5}\right\}\right),\left(e_{2},\left\{h_{3}\right\}\right),\left(e_{3},\left\{h_{1}, h_{3}\right\}\right),\left(e_{4}, \varnothing\right),\left(e_{5},\left\{h_{6}\right\}\right)\right\}, \\
& G_{4 E}=\left\{\left(e_{1},\left\{h_{2}\right\}\right),\left(e_{2},\left\{h_{4}\right\}\right),\left(e_{3},\left\{h_{1}, h_{2}, h_{4}\right\}\right),\left(e_{4}, \varnothing\right),\left(e_{5}, \varnothing\right)\right\}, \\
& G_{5 E}=\left\{\left(e_{1},\left\{h_{4}, h_{5}\right\}\right),\left(e_{2},\left\{h_{1}\right\}\right),\left(e_{3},\left\{h_{4}\right\}\right),\left(e_{4},\left\{h_{5}\right\}\right),\left(e_{5},\left\{h_{4}, h_{5}\right\}\right)\right\}, \\
& G_{6 E}=\left\{\left(e_{1},\left\{h_{6}, h_{7}\right\}\right),\left(e_{2},\left\{h_{2}, h_{3}\right\}\right),\left(e_{3},\left\{h_{2}, h_{3}, h_{7}\right\}\right),\left(e_{4},\left\{h_{6}, h_{7}\right\}\right),\left(e_{5}, \varnothing\right)\right\}, \\
& G_{7 E}=\left\{\left(e_{1},\left\{h_{2}, h_{4}, h_{7}\right\}\right),\left(e_{2},\left\{h_{4}, h_{7}\right\}\right),\left(e_{3},\left\{h_{1}, h_{5}\right\}\right),\left(e_{4}, \varnothing\right),\left(e_{5}, \varnothing\right)\right\} .
\end{aligned}
$$

(4) The soft weak structure of programmes of trip proposed by tourism company $\mathrm{D}$ (given in Table 4 ) is
$\tau_{D}=\left\{\widetilde{\Phi}, t G_{i E} n: q i h={ }_{1}, 2 x, 7 \ldots C, ; 7\right\}$, where $G_{i E}$ is given by 
TABLE 2: Trip programmes of tourism company B.

\begin{tabular}{|c|c|c|c|c|c|}
\hline \multirow{2}{*}{$X$} & \multicolumn{5}{|c|}{ E } \\
\hline & $e_{1}:$ accommodation & $e_{2}$ : eating & $e_{3}:$ cinema & $e_{4}:$ swimming & $e_{5}:$ celebrations \\
\hline Saturday & $\left\{h_{1}, h_{2}\right\}$ & $\left\{h_{3}\right\}$ & $\left\{h_{1}, h_{2}\right\}$ & $\varnothing$ & $\varnothing$ \\
\hline Sunday & $\left\{h_{7}\right\}$ & $\left\{h_{7}\right\}$ & $\varnothing$ & $\left\{h_{1}, h_{4}, h_{6}\right\}$ & $\left\{h_{1}, h_{4}, h_{6}\right\}$ \\
\hline Monday & $\left\{h_{3}, h_{4}\right\}$ & $\left\{h_{1}, h_{2}\right\}$ & $\left\{h_{1}, h_{3}\right\}$ & $\left\{h_{3}, h_{4}, h_{6}\right\}$ & $\varnothing$ \\
\hline Tuesday & $\left\{h_{2}, h_{3}\right\}$ & $\left\{h_{4}\right\}$ & $\left\{h_{1}, h_{4}, h_{5}\right\}$ & $\left\{h_{2}\right\}$ & $\varnothing$ \\
\hline Wednesday & $\left\{h_{2}, h_{4}, h_{6}\right\}$ & $\left\{h_{1}\right\}$ & $\left\{h_{7}\right\}$ & $\left\{h_{3}, h_{5}\right\}$ & $\left\{h_{5}, h_{6}, h_{7}\right\}$ \\
\hline Thursday & $\left\{h_{3}\right\}$ & $\left\{h_{7}\right\}$ & $\varnothing$ & $\left\{h_{1}, h_{6}\right\}$ & $\left\{h_{4}, h_{5}, h_{7}\right\}$ \\
\hline Friday & $\left\{h_{1}, h_{4}, h_{5}\right\}$ & $\left\{h_{1}, h_{4}, h_{5}\right\}$ & $\varnothing$ & $\left\{h_{7}\right\}$ & $\varnothing$ \\
\hline
\end{tabular}

TABle 3: Trip programmes of tourism company C.

\begin{tabular}{|c|c|c|c|c|c|}
\hline \multirow{2}{*}{$X$} & \multicolumn{5}{|c|}{$E$} \\
\hline & $e_{1}:$ accommodation & $e_{2}$ : eating & $e_{3}:$ cinema & $e_{4}:$ swimming & $e_{5}:$ celebrations \\
\hline Saturday & $\left\{h_{1}\right\}$ & $\left\{h_{1}, h_{3}\right\}$ & $\left\{h_{2}, h_{3}\right\}$ & $\varnothing$ & $\varnothing$ \\
\hline Sunday & $\left\{h_{6}, h_{7}\right\}$ & $\left\{h_{4}, h_{7}\right\}$ & $\varnothing$ & $\left\{h_{4}, h_{5}\right\}$ & $\left\{h_{4}, h_{5}\right\}$ \\
\hline Monday & $\left\{h_{1}, h_{2}, h_{5}\right\}$ & $\left\{h_{3}\right\}$ & $\left\{h_{1}, h_{3}\right\}$ & $\varnothing$ & $\left\{h_{6}\right\}$ \\
\hline Tuesday & $\left\{h_{2}\right\}$ & $\left\{h_{4}\right\}$ & $\left\{h_{1}, h_{2}, h_{4}\right\}$ & $\varnothing$ & $\varnothing$ \\
\hline Wednesday & $\left\{h_{4}, h_{5}\right\}$ & $\left\{h_{1}\right\}$ & $\left\{h_{4}\right\}$ & $\left\{h_{5}\right\}$ & $\left\{h_{4}, h_{5}\right\}$ \\
\hline Thursday & $\left\{h_{6}, h_{7}\right\}$ & $\left\{h_{2}, h_{3}\right\}$ & $\left\{h_{2}, h_{3}, h_{7}\right\}$ & $\left\{h_{6}, h_{7}\right\}$ & $\varnothing$ \\
\hline Friday & $\left\{h_{2}, h_{4}, h_{7}\right\}$ & $\left\{h_{4}, h_{7}\right\}$ & $\left\{h_{1}, h_{5}\right\}$ & $\varnothing$ & $\varnothing$ \\
\hline
\end{tabular}

TABLE 4: Trip programmes of tourism company D.

\begin{tabular}{|c|c|c|c|c|c|}
\hline \multirow{2}{*}{$X$} & \multicolumn{5}{|c|}{$E$} \\
\hline & $e_{1}:$ accommodation & $e_{2}$ : eating & $e_{3}:$ cinema & $e_{4}:$ swimming & $e_{5}:$ celebrations \\
\hline Saturday & $\left\{h_{4}, h_{5}, h_{7}\right\}$ & $\left\{h_{4}, h_{5}, h_{7}\right\}$ & $\left\{h_{4}, h_{5}, h_{7}\right\}$ & $\varnothing$ & $\varnothing$ \\
\hline Sunday & $\left\{h_{1}, h_{6}\right\}$ & $\left\{h_{3}\right\}$ & $\left\{h_{2}\right\}$ & $\left\{h_{1}, h_{2}, h_{6}\right\}$ & $\varnothing$ \\
\hline Monday & $\left\{h_{6}\right\}$ & $\left\{h_{3}\right\}$ & $\left\{h_{1}, h_{6}\right\}$ & $\left\{h_{3}, h_{5}\right\}$ & $\left\{h_{7}\right\}$ \\
\hline Tuesday & $\left\{h_{3}, h_{5}, h_{7}\right\}$ & $\left\{h_{3}, h_{6}\right\}$ & $\left\{h_{3}\right\}$ & $\left\{h_{4}, h_{5}\right\}$ & $\left\{h_{3}, h_{5}\right\}$ \\
\hline Wednesday & $\left\{h_{2}, h_{5}\right\}$ & $\left\{h_{2}, h_{5}\right\}$ & $\varnothing$ & $\left\{h_{1}, h_{2}, h_{4}\right\}$ & $\varnothing$ \\
\hline Thursday & $\left\{h_{4}, h_{7}\right\}$ & $\left\{h_{2}, h_{5}\right\}$ & $\varnothing$ & $\varnothing$ & $\left\{h_{6}, h_{7}\right\}$ \\
\hline Friday & $\left\{h_{1}, h_{2}\right\}$ & $\left\{h_{3}\right\}$ & $\left\{h_{4}\right\}$ & $\left\{h_{3}, h_{7}\right\}$ & $\varnothing$ \\
\hline
\end{tabular}

$$
\begin{aligned}
& G_{1 E}=\left\{\left(e_{1},\left\{h_{4}, h_{5}, h_{7}\right\}\right),\left(e_{2},\left\{h_{4}, h_{5}, h_{7}\right\}\right),\left(e_{3},\left\{h_{4}, h_{5}, h_{7}\right\}\right),\left(e_{4}, \varnothing\right),\left(e_{5}, \varnothing\right)\right\}, \\
& G_{2 E}=\left\{\left(e_{1},\left\{h_{1}, h_{6}\right\}\right),\left(e_{2},\left\{h_{3}\right\}\right),\left(e_{3},\left\{h_{2}\right\}\right),\left(e_{4},\left\{h_{1}, h_{2}, h_{6}\right\}\right),\left(e_{5}, \varnothing\right)\right\}, \\
& G_{3 E}=\left\{\left(e_{1},\left\{h_{6}\right\}\right),\left(e_{2},\left\{h_{3}\right\}\right),\left(e_{3},\left\{h_{1}, h_{6}\right\}\right),\left(e_{4},\left\{h_{3}, h_{5}\right\}\right),\left(e_{5},\left\{h_{7}\right\}\right)\right\}, \\
& G_{4 E}=\left\{\left(e_{1},\left\{h_{3}, h_{5}, h_{7}\right\}\right),\left(e_{2},\left\{h_{3}, h_{6}\right\}\right),\left(e_{3},\left\{h_{3}\right\}\right),\left(e_{4},\left\{h_{4}, h_{5}\right\}\right),\left(e_{5},\left\{h_{3}, h_{5}\right\}\right)\right\}, \\
& G_{5 E}=\left\{\left(e_{1},\left\{h_{2}, h_{5}\right\}\right),\left(e_{2},\left\{h_{2}, h_{5}\right\}\right),\left(e_{3}, \varnothing\right),\left(e_{4},\left\{h_{1}, h_{2}, h_{4}\right\}\right),\left(e_{5}, \varnothing\right)\right\}, \\
& G_{6 E}=\left\{\left(e_{1},\left\{h_{4}, h_{7}\right\}\right),\left(e_{2},\left\{h_{2}, h_{5}\right\}\right),\left(e_{3}, \varnothing\right),\left(e_{4}, \varnothing\right),\left(e_{5},\left\{h_{6}, h_{7}\right\}\right)\right\}, \\
& G_{7 E}=\left\{\left(e_{1},\left\{h_{1}, h_{2}\right\}\right),\left(e_{2},\left\{h_{3}\right\}\right),\left(e_{3},\left\{h_{4}\right\}\right),\left(e_{4},\left\{h_{3}, h_{7}\right\}\right),\left(e_{5}, \varnothing\right)\right\} .
\end{aligned}
$$

Now, we analyze the four programmes of trips with respect to soft separation axioms as follows.

(1) A soft weak structure $\left(X, \tau_{A}, E\right)$ of tourism company A is non-pt-soft $\mathscr{W} T_{0}$ because $h_{2} \neq h_{5}$ and there does not exist a $\mathscr{W}$-soft open subset $G_{E}$ of $\left(X, \tau_{A}, E\right)$ such that $h_{2}$ partially belongs to $G_{E}$ and $h_{5}$ does not totally belong to it or $h_{5}$ partially belongs to $G_{E}$ and $h_{2}$ does not totally belong to it.
(2) It can be checked that a soft weak structure $\left(X, \tau_{B}, E\right)$ of tourism company B is $p t$-soft $\mathscr{W} T_{0}$. On the other hand, it is not $p t$-soft $\mathscr{W} T_{1}$ because $h_{2} \neq h_{3}$ and there does not exist a $\mathscr{W}$-soft open subset $G_{E}$ of $\left(X, \tau_{A}, E\right)$ such that $h_{2}$ partially belongs to $G_{E}$ and $h_{3}$ does not totally belong to it.

(3) It can be checked that a soft weak structure $\left(X, \tau_{C}, E\right)$ of tourism company $\mathrm{C}$ is $p t$-soft $\mathscr{W} T_{0}$. On the other 
hand, it is not $p t$-soft $\mathscr{W} T_{1}$ because $h_{2} \neq h_{3}$ and there does not exist a $\mathscr{W}$-soft open subset $G_{E}$ of $\left(X, \tau_{A}, E\right)$ such that $h_{3}$ partially belongs to $G_{E}$ and $h_{2}$ does not totally belong to it.

(4) It can be checked that a soft weak structure $\left(X, \tau_{D}, E\right)$ of tourism company $\mathrm{D}$ is $p t$-soft $\mathscr{W} T_{1}$. On the other hand, it is not $p t$-soft $\mathscr{W} T_{2}$ because $h_{1} \neq h_{2}$ and there do not exist disjoint $\mathscr{W}$-soft open subsets $G_{1 E}$ and $G_{2 E}$ of $\left(X, \tau_{A}, E\right)$ partially containing $h_{1}$ and $h_{2}$, respectively, such that $h_{2}$ does not totally belong to $G_{1 E}$ and $h_{1}$ does not totally belong to $G_{2 E}$.

According to the data given above and their analysis, we infer that the offer presented by tourism company $\mathrm{D}$ is more convenient (optimal choice) for the customers who belong to the group of many visited places without repetition as much as possible. However, we infer that the optimal offers presented by tourism company $\mathrm{B}$ and tourism company $\mathrm{C}$ are more convenient (optimal choices) for the customers who belong to the group of few visited places with repetition as much as possible.

In what follows, we present an algorithm showing the method of selecting the optimal offers.

(1) Select the desired location of trip.

(2) Take the offering brochures given from some tourism companies.

(3) Determine the number of visited places $X=\left\{h_{i}: i=\right.$ $1,2, \ldots, n\}$ and available activities $E=\left\{e_{i}: i=1,2\right.$, $\ldots, m\}$.

(4) Write every trip programmes in a table (as given in Tables 1-4).

(5) Transfer each table in the previous step to its corresponding soft weak structure.

(6) Classify the obtained soft weak structures with respect to soft $p t$-soft $\mathscr{W} T_{i}$-spaces. In other words, determine which one is non-pt-soft $\mathscr{W} T_{0}, p t$-soft $\mathscr{W} T_{0}$, pt-soft $\mathscr{W} T_{1}$, or $p t$-soft $\mathscr{W} T_{2}$.

(7) Determine an optimal choice according to your personality characteristic: if you belong to the group of many visited places without iteration, then you will prefer tourism company which has trip programmes satisfying strong form of available $p t$-soft $\mathscr{W} T_{i}$-space. In contrast, If you belong to the group of few visited places, then you will prefer tourism company which has trip programmes satisfying weak form of available $p t$-soft $\mathscr{W} T_{i}$-space.

(8) If there is more than one optimal choice, then you can select any one of them satisfying his or her option.

Last but not least, we recommend the tourism companies to take into consideration the criteria proposed in this study when they prepare tourism programmes as they can enlist experts in this field and benefit from their experience to raise the standard of turnout.

\section{Discussion}

The method followed in the previous application is based on $p t$-soft $\mathscr{W} T_{1}$-spaces $(i=0,1,2)$ which are defined in this study. This method relies on two factors, the first one comes from the classification induced from soft separation axioms and the second one comes from the personality characteristics of the customers.

One of the advantages of this technique is the relaxation of conditions of some structures such as soft topology, supra soft topology, and generalized soft topology. In other words, we do not need to check the finite soft union. We write a finite case instead of arbitrary case for a soft union because we deal with a system consisting of finite elements and attributes and intersection which gives us freedom and ease to model the phenomena under study. Another merit of this technique is the nature (type) of the belong and nonbelong relations that are utilized to define those types of soft separation axioms. These relations (partial belong and total nonbelong relations which are the core of our approach in this manuscript) offer multiple options to "transfer" the reallife problem to a mathematical model compared with their counterparts of soft separation axioms using total belong and total nonbelong relations introduced in [22].

On the other hand, there are some limitations of our method with the number of variables (which in this study are the days, places, and activities). According to our application, we should examine $\left(\begin{array}{l}7 \\ 2\end{array}\right)=21$ different cases of places $h_{i}$. In this case, the total relation whether it is belonging or nonbelonging requires more soft sets to satisfy the different cases. This implies that modeling under total belong or total nonbelong hampers the description of the phenomenon; consequentially, the flexibility (completeness and accuracy) of this method to model a phenomenon is less than that of a method induced from partial belong and partial nonbelong relations given in [24]. We conclude that we can represent the phenomena using partial belong and partial nonbelong relations more easier than using partial belong and total nonbelong relations.

It noteworthy that the conditions regarding the number of variables in our approach will be similar to the required conditions in the case of total belong and partial nonbelong relations given in [23]. The differences are induced by replacing the total nonbelong relation $\notin$ by the total belong relation $\epsilon$.

Finally, we can apply this method, taking into account the nature of each phenomenon, in the engineering sciences as follows.

(1) Decision making and topology have a long joint tradition since the modern statement of the classical Weierstrass extreme value theorem. It combines two topological concepts called continuity of a realvalued function and compactness of the domain (both with respect to a given topology). They represent a necessary and sufficient condition to guarantee the existence of the maximum and minimum values of the function. The success of this 
technique was amplified by its adoption in fields like engineering sciences, computer sciences, and mathematical economics. This matter can be adopted on the version of soft setting by replacing the classical notions (compactness, function, and real numbers) by their soft counterparts (soft compactness, soft function, and soft real numbers).

(2) Some practical experiments in the civil engineering require classification of the materials according to their characteristics (attribute set or parameter set $E$ ) which can be expressed using the concept of soft sets. Then, we study the separation of them with respect to the group of soft sets which are constructed from the practical experiments. In this group of soft sets, we add the absolute and null soft sets to initiate a soft weak structure. Finally, we determine the type of this soft weak structure with respect to $p t$-soft $\mathscr{W} T_{i}(i=$ $0,1,2)$ or non-pt-soft $\mathscr{W} T_{0}$.

(3) The researchers in the communication engineering endeavor to select the best protocol to solve the noisy problems in wireless networks. They evaluate the performance of these protocols according to the proposed scenarios. We plan with some engineers to propose some protocols using the appropriate soft structure and compare with those proposed [46] to select the optimal protocol to solve the interference problems in wireless networks.

\section{Conclusion}

In this study, we have obtained a new class of soft topological spaces by defining the concepts of $p t$-soft $\alpha T_{i}$-spaces $(i=0,1,2,3,4)$. They are formulated with respect to partial belong and total nonbelong relations between ordinary points and soft $\alpha$-open sets. We have investigated the interrelations between these concepts and their parametric topological spaces. Some illustrative examples are given to clarify the obtained relationships and results. In the end, we have defined $p t$-soft $T_{i}$-spaces $(i=0,1,2)$ on a soft weak structure and applied them in solving a decision-making problem. In this regard, we have proposed an algorithm of an optimal selection and provided a real example to explain how this algorithm works. It is worthily noted that this paper is the first emergence of real applications of soft separation axioms in decision-making problems.

As future works, we shall study these concepts with respect to another generalizations of soft open sets such as soft preopen and soft semiopen sets. Also, we shall redefine these concepts using partial belong and partial nonbelong relations and investigate their characterizations. Moreover, we will study them on the contents of supra soft topological spaces, minimal soft topological spaces, and soft weak structures. In addition, we attempt to apply these concepts in the areas of engineering sciences and computer sciences (as we explained in Discussion section).

In conclusion, we hope that the initiated notions will be beneficial for researchers and scholars to promote and progress the study in soft topology and decision-making problems.

\section{Data Availability}

No data were used to support this study.

\section{Conflicts of Interest}

The author declares that there are no conflicts of interest.

\section{References}

[1] D. Molodtsov, "Soft set theory-first results," Computers \& Mathematics with Applications, vol. 37, no. 4-5, pp. 19-31, 1999.

[2] P. K. Maji, R. Biswas, and A. R. Roy, "Soft set theory," Computers \& Mathematics with Applications, vol. 45, no. 4-5, pp. 555-562, 2003.

[3] M. I. Ali, F. Feng, X. Liu, W. K. Min, and M. Shabir, "On some new operations in soft set theory," Computers \& Mathematics with Applications, vol. 57, no. 9, pp. 1547-1553, 2009.

[4] K. V. Babitha and J. J. Sunil, "Soft set relations and functions," Computers \& Mathematics with Applications, vol. 60, no. 7, pp. 1840-1849, 2010.

[5] K. Qin and Z. Hong, "On soft equality," Journal of Computational and Applied Mathematics, vol. 234, no. 5, pp. 1347$1355,2010$.

[6] T. M. Al-shami, "Investigation and corrigendum to some results related to g-soft equality and $g$-soft equality relations," Filomat, vol. 33, no. 11, pp. 3375-3383, 2019.

[7] T. M. Al-shami and M. E. El-Shafei, "T-soft equality relation," Turkish Journal of Mathematics, vol. 44, no. 4, pp. 1427-1441, 2020.

[8] M. Ali, H. Khan, L. H. Son, F. Smarandache, and W. B. V. Kandasamy, "New soft sets based class of linear algebraic codes," Symmetry, vol. 10, pp. 1-10, 2018.

[9] N. Çağman and S. Enginoglu, "Soft matrix theory and its decision making," Computers and Mathematics with Applications, vol. 59, pp. 3308-3314, 2010.

[10] F. Karaaslan, "Soft classes and soft rough classes with applications in decision making," Mathematical Problems in Engineering, vol. 2016, p. 11, Article ID 1584528, 2016.

[11] S. Yuksel, T. Dizman, G. Yildizdan, and U. Sert, "Application of soft sets to diagnose the prostate cancer risk," Journal of Inequalities and Applications, vol. 2013, p. 229, 2013.

[12] M. Shabir and M. Naz, "On soft topological spaces," Computers \& Mathematics with Applications, vol. 61, no. 7, pp. 1786-1799, 2011.

[13] N. Çağman, S. Karataş, and S. Enginoglu, "Soft topology," Computers and Mathematics with Applications, vol. 62, pp. 351-358, 2011.

[14] A. Aygünoğlu and H. Aygün, "Some notes on soft topological spaces," Neural Computing and Applications, vol. 21, pp. 113-119, 2012.

[15] T. Hida, "A comprasion of two formulations of soft compactness," Annals of Fuzzy Mathematics and Informatics, vol. 8, no. 4, pp. 511-524, 2014.

[16] T. M. Al-shami, M. E. El-Shafei, and M. Abo-Elhamayel, "Almost soft compact and approximately soft Lindelöf spaces," Journal of Taibah University for Science, vol. 12, no. 5, pp. 620-630, 2018.

[17] T. M. Al-shami and L. D. R. Kočinac, "Nearly soft Menger spaces," Journal of Mathematics, vol. 2020, p. 9, Article ID 3807418, 2020. 
[18] K. V. Babitha and S. J. John, "Studies on soft topological spaces," Journal of Intelligent \& Fuzzy Systems, vol. 28, no. 4, pp. 1713-1722, 2015.

[19] K. V. Babitha and J. J. Sunil, "Soft topologies generated by soft set relations," in Handbook of Research on Generalized and Hybrid Set Structures and Applications for Soft Computing, pp. 118-126, IGI Global Pub, Hershey, PA, USA, 2015.

[20] A. Kharal and B. Ahmad, "Mappings on soft classes," New Mathematics and Natural Computation, vol. 7, no. 3, pp. 471-481, 2011.

[21] D. Wardowski, "On a soft mapping and its fixed points," Fixed Point Theory Application, vol. 2013, p. 182, 2013.

[22] M. E. El-Shafei, M. Abo-Elhamayel, and T. M. Al-shami, "Partial soft separation axioms and soft compact spaces," Filomat, vol. 32, no. 13, pp. 4755-4771, 2018.

[23] T. M. Al-shami and M. E. El-Shafei, "Partial belong relation on soft separation axioms and decision-making problem, two birds with one stone," Soft Computing, vol. 24, no. 7, pp. 5377-5387, 2020.

[24] M. E. El-Shafei and T. M. Al-shami, "Applications of partial belong and total non-belong relations on soft separation axioms and decision-making problem," Journal of Computational and Applied Mathematics, vol. 39, p. 138, 2020.

[25] S. Bayramov and C. G. Aras, "A new approach to separability and compactness in soft topological spaces," TWMS Journal of Applied and Engineering Mathematics, vol. 9, no. 1, pp. 82-93, 2018.

[26] O. Tantawy, S. A. El-Sheikh, and S. Hamde, "Separation axioms on soft topological spaces," Annals of Fuzzy Mathematics and Informatics, vol. 11, no. 4, pp. 511-525, 2016.

[27] A. Singh and N. S. Noorie, "Remarks on soft axioms," Annals of Fuzzy Mathematics and Informatics, vol. 14, no. 5, pp. 503-513, 2017.

[28] M. Terepeta, "On separating axioms and similarity of soft topological spaces," Soft Computing, vol. 23, no. 3, pp. 1049-1057, 2019.

[29] J. C. R. Alcantud, "Soft open bases and a novel construction of soft topologies from bases for topologies," Mathematics, vol. 8, no. 5, p. 672, 2020.

[30] T. M. Al-shami, "Comments on "Soft mappings spaces," The Scientific World Journal, vol. 2019, p. 2, Article ID 6903809, 2019.

[31] T. M. Al-shami, "Comments on some results related to soft separation axioms," Afrika Matematika, vol. 31, no. 7-8, pp. 1105-1119, 2020.

[32] S. J. John, Soft Sets: Theory and Applications, Springer, Berlin, Germany, 2021.

[33] S. Ashraf, S. Abdullah, and L. Abdullah, "Child development influence environmental factors determined using spherical fuzzy distance measures," Mathematics, vol. 7, no. 8, p. 661, 2019.

[34] S. Ashraf, S. Abdullah, T. Mahmood, F. Ghani, and T. Mahmood, "Spherical fuzzy sets and their applications in multi-attribute decision making problems," Journal of Intelligent \& Fuzzy Systems, vol. 36, no. 3, p. 2829, 2019.

[35] S. Ashraf, T. Mahmood, S. Abdullah, and Q. Khan, "Different approaches to multi-criteria group decision making problems for picture fuzzy environment," Bulletin of the Brazilian Mathematical Society, New Series, vol. 50, no. 2, pp. 373-397, 2019.

[36] F. Feng, C. Li, B. Davvaz, and M. I. Ali, "Soft sets combined with fuzzy sets and rough sets: a tentative approach," Soft Computing, vol. 14, no. 9, pp. 899-911, 2010.
[37] S. Das and S. K. Samanta, "Soft metric," Annals of Fuzzy Mathematics and Informatics, vol. 6, no. 1, pp. 77-94, 2013.

[38] S. Nazmul and S. K. Samanta, "Neighbourhood properties of soft topological spaces," Annals of Fuzzy Mathematics and Informatics, vol. 6, no. 1, pp. 1-15, 2013.

[39] M. Akdag and A. Ozkan, "Soft $\alpha$-open sets and soft $\alpha$-continuous functions $\alpha$-open sets and soft $\alpha$-continuous functions," Abstract and Applied Analysis, vol. 2014, Article ID 891341, 7 pages, 2014.

[40] M. Akdag and A. Ozkan, "On soft $\alpha$-separation axioms," Journal of Advanced Studies in Topology, vol. 5, no. 4, pp. 16-24, 2014.

[41] T. M. Al-shami and E. A. Abo-Tabl, Soft $\alpha$-separation Axioms and $\alpha$-Fixed Soft Points.

[42] T. M. Al-shami and L. D. R. Kočinac, "The equivalence between the enriched and extended soft topologies," Journal of Computational and Applied Mathematics, vol. 18, no. 2, pp. 149-162, 2019.

[43] T. M. Al-shami, L. D. R. Kočinac, and B. A. Asaad, "Sum of soft topological spaces," Mathematics, vol. 8, no. 6, p. 990, 2020.

[44] I. Zorlutuna, M. Akdag, W. K. Min, and S. Atmaca, "Remarks on soft topological spaces," Annals of Fuzzy Mathematics and Informatics, vol. 2, pp. 171-185, 2012.

[45] A. H. Zakari, A. Ghareeb, and S. Omran, "On soft weak structures," Soft Computing, vol. 21, no. 10, pp. 2553-2559, 2017.

[46] W. M. Bahgat, M. M. Al-dabaa, and H. H. Soliman, "Performance evaluation of cooperative relaying for MAC protocols," International Journal of Computer Applications, vol. 158, no. 1, pp. 35-49, 2017. 